\title{
Differences in the bacterial profiles and physicochemical between natural and inoculated fermentation of vegetables from Shanxi Province
}

Zhidi Chen ${ }^{1 \dagger}$, Jianyi Kang ${ }^{1,2+}$, Yao Zhang ${ }^{1 \dagger}$, Xinxin $\mathrm{Yi}^{1}$, Xiaona Pang ${ }^{1}$, Hongmei Li-Byarlay $^{3}$ and Xiuzhi Gao ${ }^{1 *}$

\begin{abstract}
Purpose: Fermented vegetables can be divided into two types, natural fermented and artificially inoculated fermented. By detecting and identifying the changes of bacterial diversity using physical and chemical indicators during natural and inoculation fermentation, we analyzed and determined the dominant bacteria in the fermentation process and revealed the relationship between bacteria and volatile substances.

Methods: We used the Illumina Miseq to sequence the bacteria in fermented vegetable samples at different fermentation periods, and calculated the total number of mesophilic microorganisms and lactic acid bacteria. We used the $\mathrm{pH}$ and nitrite to monitor the acidification process. GC-MS was used to determine volatile flavor compounds. Finally, we analyzed the correlation between volatile flavor compounds and bacteria.

Results: Total mesophilic microorganisms and the number of lactic acid bacteria in the inoculated fermentation were higher than the natural fermentation. The bacterial diversity Shannon and Simpson indexes of the natural fermentation, higher than those of inoculated fermentation in $0 \sim 7$ days, were between $55 \sim 71 \%$ and 36 45\%, respectively. On the 7th day, the proportion of Lactobacillus in the natural fermentation and inoculated fermentation were $53.4 \%$ and $90.2 \%$, respectively, which were significantly different. Lactobacillus was the dominant genus in the fermented vegetables and an important genus to promote the formation of volatile flavors. Lactobacillus was negatively correlated with two volatile substances (4-[2,2,6-trimethyl-7-oxabicyclo [4.1.0] hept-1-yl]3-Buten-2-one (K4) and a-Phellandrene (X1)) and played a leading role in the fermentation process.

Conclusions: Results demonstrated that the total number of mesophilic microorganisms and lactic acid bacteria in inoculated fermentation were more than those in natural fermentation. Inoculated fermentation can shorten the fermentation cycle and reduce the content of nitrite. Lactic acid bacteria were the dominant bacteria in fermented vegetables.
\end{abstract}

Keywords: Natural fermentation, Inoculated fermentation, High-throughput sequencing, Bacterial diversity, Correlation Analysis

\footnotetext{
* Correspondence: gxz@bua.edu.cn

'Zhidi Chen, Jianyi Kang, and Yao Zhang contributed equally to this work.

'Beijing Laboratory of Food Quality and Safety, Beijing Key Laboratory of

Agricultural Product Detection and Control of Spoilage Organisms and

Pesticide Residue, Beijing Engineering Laboratory of Probiotics Key

Technology Development, Beijing University of Agriculture, Beijing 102206,

China

Full list of author information is available at the end of the article
}

(c) The Author(s). 2020 Open Access This article is licensed under a Creative Commons Attribution 4.0 International License, which permits use, sharing, adaptation, distribution and reproduction in any medium or format, as long as you give appropriate credit to the original author(s) and the source, provide a link to the Creative Commons licence, and indicate if changes were made. The images or other third party material in this article are included in the article's Creative Commons licence, unless indicated otherwise in a credit line to the material. If material is not included in the article's Creative Commons licence and your intended use is not permitted by statutory regulation or exceeds the permitted use, you will need to obtain permission directly from the copyright holder. To view a copy of this licence, visit http://creativecommons.org/licenses/by/4.0/ 


\section{Introduction}

Fermentation has been applied in food processing for millennia. Fermented foods constitute an integral part of the human diet all over the world (Tamang et al. 2016). Fermented vegetables, which are made of vegetables such as cabbage, radish, beet, celery, or cucumber with various seasonings, are generally fermented from a few days to several months.

Similar to other fermented foods such as fermented soy, yogurt, and cheese, fermented vegetables have a unique aroma, taste, and great nutritional value, which are entirely dependent upon the fermentation conditions (Mcfeeters 2004). Many clinical and animal studies have demonstrated that fermented vegetables significantly reduce the risks of obesity (Kim et al. 2011; Kwak et al. 2012), diabetes (Zhang et al. 2018), oxidation (Kim et al. 2017a), hypercholesterolemia (Jung et al. 2014a), cancer (Yeh and Yen 2005), and help to stimulate the immune system (Caggianiello et al. 2016). Fermented vegetables have been considered as one of the most nutritional and health-beneficial foods in the world (Kim et al. 2012).

The most important factor associated with its health benefits is probiotic lactic acid bacteria (LAB), including Lactobacillus strains (Xiong et al. 2013). Different LAB strains have been used in fermented vegetables, such as Lactococcus lactis WiKim0098 and Leuconostoc citreum WiKim0096 (Kim et al. 2019), Lactiplantibacillus plantarum D-103 (Lee et al. 2016), Leuconostoc mesenteroides strain B1 (Jung et al. 2012), Weissella cibaria WC018, and Lactiplantibacillus plantarum LP067 (Xiang et al. 2020).

Microbial community composition at the initial fermentation process may be related to different types of raw materials. Given that the majority of fermented vegetables include several raw ingredients (Chen et al. 2014b; Yu et al. 2012), due to the natural transfer of the various indigenous microbiota, a competitive or cooperative microbial relationships may be established during initial fermentation, and the growth of dominant microbes during late fermentation. Fermentation failure, defined as a dysbiotic microbial community in foods, may cause food spoilage followed by the outgrowth of toxic or food poisoning microbes (Wei et al. 2020). Unfavorable microbial communities might result from the decreased resistance of desirable fermentative microbes to invasion caused by undesirable microbes. In most fermented vegetables, colonization resistance is mainly conferred by the outgrowth of LAB (Jung et al. 2018). Therefore, the LAB starter was added during initial vegetable preparation can avoid fermentation failure. However, many traditional household fermented foods still rely upon the natural transfer of fermentative microbes from the raw ingredients (Jung et al. 2014b; Lee et al. 2015a; Lee et al. 2015b).
Taxonomic studies based on microbial culturedependent and independent approaches (e.g., bacterial 16S rRNA gene amplicon sequencing) showed that fermented vegetables possess a distinct microbial community (Tamang et al. 2016). LAB, including Weissella, Lactobacillus, and Leuconostoc species, are the dominant microbes in the vegetable fermentation (Jung et al. 2011; Lee et al. 2017; Park et al. 2012). The vast majority of fermented vegetable consumption in China is household fermented vegetables (Yang et al. 2020). Raw ingredientderived fermentative microbes are the main bacteria in household fermented vegetables. The direct origin sources of the fermentative microbes and their causative role(s) on microbial community assembly patterns and processes during fermented vegetable fermentation are scarcely known.

Fresh vegetables have a short storage time at room temperature, which may lead to decomposition during transportation. Fermented vegetables not only have high nutritional value but also can be stored at room temperature for a long time. This study compared the number of microorganisms, the bacterial diversity, and physicochemical indexes of Shanxi local fermented vegetables during natural fermentation (NF) and inoculated fermentation (IF). Further analysis revealed the correlation between bacterial and flavors in the fermented vegetables. LAB isolated from naturally fermented vegetables (LV02 and LV73) are suggested to be used to inoculate naturally fermented vegetables. Inoculated fermentation can improve the stability of fermented vegetable products and shorten the fermentation cycle. This study provides a foundation of knowledge for the standardized industrialization of local fermented vegetables.

\section{Materials and methods}

\section{Bacterial cultures}

Probiotic LV02 (Lactiplantibacillus plantarum V02 MH885507) and LV73 (Lentilactobacillus diolivorans V73 MH885506) isolated and identified from traditional fermented vegetables was obtained from Shanxi Province local fermented vegetables. LV02 and LV73 were deposited in the China General Microbiological Culture Collection Center (CGMCC) on November 12, 2018; the deposit number of LV02 is CGMCC No. 16727, and LV73 is CGMCC No. 16728. Strains were stored in $30 \%(\mathrm{v} / \mathrm{v})$ glycerol at $-80{ }^{\circ} \mathrm{C}$. They were inoculated into MRS (Man Rogasa Sharpe) (Land bridge, Beijing) broth and grown at $37{ }^{\circ} \mathrm{C}$ for $12 \mathrm{~h}$ to obtain a cell count of $8-9 \lg \mathrm{CFU} / \mathrm{mL}$, then used for inoculation at $0.5 \%(\mathrm{w} / \mathrm{v})$ in vegetables to prepare the starter for fermentation. All vegetables were purchased from the local supermarket. 


\section{Preparation of fermented vegetables}

The fermented vegetables were prepared following the methods in Jung et al. (2016), with slight modification. We mixed sliced cabbages $(8 \mathrm{~kg})$, celeries $(1 \mathrm{~kg})$, and carrots $(1 \mathrm{~kg})$ with $500 \mathrm{~mL}$ of $4 \%$ brine, then divided them into two equal portion groups: (1) the control fermented vegetables as natural fermentation (NF), and (2) treated group inoculated with $0.5 \%(\mathrm{w} / \mathrm{v})$ of probiotic LV02 and LV73 to prepare probiotic fermented vegetables (IF). The seasoned vegetables were fermented at 25 ${ }^{\circ} \mathrm{C}$ for 7 days in the jar. We collected $500 \mathrm{~g}$ of samples (both liquid and vegetable) each day in the polyethylene bags. The NF and IF products ( $n=3$ per product group) were stored under $-80{ }^{\circ} \mathrm{C}$ until analysis. The chemical and microbiological analyses of the two groups were determined immediately (day 0 ) and after every day until 7 days of fermentation.

\section{Enumeration of viable bacteria}

The fermented vegetable samples were cultured in PCA agar (Land bridge, Beijing) at $37{ }^{\circ} \mathrm{C}$ for $48 \mathrm{~h}$ to determine the total mesophilic microorganisms, and in MRS agar (Land bridge, Beijing) at $37{ }^{\circ} \mathrm{C}$ for $24 \mathrm{~h}$ to determine the number of LAB (Jampaphaeng et al. 2018). Viable counts were expressed as colony-forming unit (CFU) per gram of the fermented vegetable samples (Han et al. 2004). Ten times echelon dilution was used in this experiment. All the experiments were performed in triplicate and recorded as mean \pm standard deviation (SD).

\section{Extraction of microbial genome}

According to the specification of E.Z. N.A. ${ }^{\circ}$ soil kit (Omega Bio-tek, Norcross, GA, USA), the total DNA (Axygen Biosciences, Union City, CA, USA) was extracted, the concentration and purity of DNA were detected, and the quality of DNA was detected by $1 \%$ agarose gel electrophoresis. The variable region of V3V4 was amplified by PCR with $338 \mathrm{~F}$ ( $5^{\prime}$ undefined ACTCCTACGGAGGAGCAGCAGCAGCAGCAG 3' undefined) primers and $806 \mathrm{R}$ ( $5^{\prime}$ undefined GGACTA CHVGGGTCTAAT 3' undefined) primers. Primers were purchased from Sangon Biotech Co., Ltd., Beijing, China.

The amplification procedure was as follows: predenaturation at $95{ }^{\circ} \mathrm{C}$ for $3 \mathrm{~min}, 27$ cycles (denaturation at $95{ }^{\circ} \mathrm{C}$ for $30 \mathrm{~s}$, annealing at $55{ }^{\circ} \mathrm{C}$ for $30 \mathrm{~s}$, extension at $72{ }^{\circ} \mathrm{C}$ for $30 \mathrm{~s}$ ), and extension of $10 \mathrm{~min}$ at $72{ }^{\circ} \mathrm{C}$.

The amplification system was $20 \mu \mathrm{L}, 4 \mu \mathrm{L} 5 \times$ FastPfu buffer, $2 \mu \mathrm{L} 2.5 \mathrm{mM}$ dNTPs, $0.8 \mu \mathrm{L}$ primer $(5 \mu \mathrm{M}), 0.4$ $\mu \mathrm{L}$ FastPfu polymerase, 10 ng DNA template.

\section{High-throughput sequencing}

PCR products were recovered by $2 \%$ agarose gel, purified, eluted by Tris- $\mathrm{HCl}$, and detected by $2 \%$ agarose gel electrophoresis. According to Illumina MiSeq Miseq PE300 Illumina, San Diego, USA, platform for sequencing (Shanghai Meiji Biopharmaceutical Technology Co., Ltd.). The original data has been uploaded to the NCBI database.

\section{Bioinformatics analysis}

Original sequencing sequence used Trimmomatic software quality control, used FLASH software for splicing. The UPARSE software was used to cluster the sequences with OTU according to $97 \%$ similarity, and removed the single sequence and chimera in the process of clustering, and annotated the species classification of each sequence. The Silva database (SSU123) was compared and the threshold value of the ratio was set to $70 \%$.

\section{Acidification process monitoring}

Production of total acid in the fermentation process of the samples was expressed by measuring changes of $\mathrm{pH}$; the $\mathrm{pH}$ level of the samples was determined with a $\mathrm{pH}$ meter (pH-250L, ISTEK, Seoul, Korea) (Rao et al. 2020).

Determination of nitrite content was according to the method by Khan et al. (2018), with some modifications. Then, $12.5 \mathrm{~mL}$ of $50 \mathrm{~g} / \mathrm{L}$ saturated borax solution was added to a beaker with $5 \mathrm{~g}$ (accurate to $0.01 \mathrm{~g}$ ) uniform samples; the contents were diluted with water. Heated in boiling water bath for $15 \mathrm{~min}$, removed and cooled to room temperature. Then $5.0 \mathrm{~mL}$ of $106 \mathrm{~g} / \mathrm{L}$ potassium ferrocyanide and $5.0 \mathrm{~mL}$ of $220 \mathrm{~g} / \mathrm{L}$ zinc acetate solution were added and the solution was shaken thoroughly and the contents were diluted to the mark $(500 \mathrm{~mL})$ with distilled water, mixed well, and left for $30 \mathrm{~min}$. Further, $40 \mathrm{~mL}$ of extracted and filtered sample solutions was removed into a $50 \mathrm{~mL}$ colorimetric tube with stopper; 2.0 $\mathrm{mL}$ of $4 \mathrm{~g} / \mathrm{L}$ paminobenzenesulfonic acid solution was added, mixed well after, and left for 3-5 min; and 1.0 $\mathrm{mL}$ of $2 \mathrm{~g} / \mathrm{L}$ naphthalene ethylenediamine hydrochloride solution was added and the contents were diluted to the mark with distilled water, mixed well, and left for 15 min; blank reagent was used as a control. Absorbance was measured at wavelength of maximum absorption $(538 \mathrm{~nm})$ against the corresponding reagent blank using single beam UV-Visible spectrophotometer. The amount of nitrite present in the unknown solution was computed from the calibration graph.

\section{SPME-GC/MS profiling}

Extraction of volatile flavor substances was examined according to the method described by $\mathrm{He}$ et al. (2020), with slight modifications. Weigh accurately $5 \mathrm{~g}$ of the cut vegetables from the fermentation process into a 20 
$\mathrm{mL}$ headspace vial and cap it. Pre-equilibrate the headspace bottle containing the sample in a thermostatic water bath at $85{ }^{\circ} \mathrm{C}$ for $5 \mathrm{~min}$, and then insert the aged $50 / 30 \mu \mathrm{m}$ diethylbenzene/carbon molecular sieve/polydimethy lsiloxane (DVB/CAR/PDMS) After the extraction head continued to equilibrate and enrich for volatile matter for $30 \mathrm{~min}$, the fiber head was retracted, the extraction head was pulled out, and the extraction head was reinserted into the GC-MS (7980A-5975C, US, Agilent Technologies Inc.) manual inlet for $5 \mathrm{~min}$ at $250{ }^{\circ} \mathrm{C}$.

\section{GC-MS analysis}

Column DB-5MS $(30 \mathrm{~m} \times 0.32 \mathrm{~mm} \times 0.25 \mu \mathrm{m})$; splitless injection mode; carrier gas: He; carrier gas flow: $1 \mathrm{~mL} /$ min; inlet temperature: $250{ }^{\circ} \mathrm{C}$; column temperature program temperature rise: $40{ }^{\circ} \mathrm{C}$ for $3 \mathrm{~min}, 5^{\circ} \mathrm{C} / \mathrm{min}$ linearly increased to $150{ }^{\circ} \mathrm{C}, 10{ }^{\circ} \mathrm{C} / \mathrm{min}$ linearly increased to 250 ${ }^{\circ} \mathrm{C}$, and then maintained at $250{ }^{\circ} \mathrm{C}$ for $5 \mathrm{~min}$. MS conditions: electron ion source (EI), electron energy $70 \mathrm{eV}$, ion source temperature $220{ }^{\circ} \mathrm{C}$, transmission line temperature $280{ }^{\circ} \mathrm{C}$; use full-scan mode to acquire signals, scanning range $50-1000 \mathrm{amu}$; ionization voltage is $70 \mathrm{eV}$.

\section{Data analysis}

The data were statistically analyzed with Excel 2007, SPSS 18 software, and compared with Duncan undefined method. The data obtained by GC-MS was compared using the NIST08 library (matching degree $>80 \%$ ); the obtained data was imported into SIMCA-P 14.0 for correlation analysis using PLS-DA. All the experiments were repeated three times, and the results were expressed by mean \pm standard deviation.

\section{Results}

\section{Evolution of inoculum}

Change of bacterial viable counts in the samples of NF and IF throughout the 7 days of storage at $25{ }^{\circ} \mathrm{C}$ was shown in the Fig. 1. The total mesophilic microorganisms and the number of LAB both showed an increasing trend in the early stage of both fermentations. The initial number of total mesophilic microorganisms in NF and IF were $4.76 \mathrm{lg} \mathrm{CFU} / \mathrm{g}$ and $5.38 \mathrm{lg} \mathrm{CFU} / \mathrm{g}$, respectively, and the $\mathrm{LAB}$ contents were $4.22 \mathrm{lg} \mathrm{CFU} / \mathrm{g}$ and $5.31 \mathrm{lg}$ $\mathrm{CFU} / \mathrm{g}$, respectively. The number of total mesophilic microorganisms and LAB in the IF vegetables was slightly higher than that of NF due to the difference in fermentation methods and individual vegetables. On the second day, the number of total mesophilic microorganisms reached the highest were $8.21 \mathrm{lg} \mathrm{CFU} / \mathrm{g}$ and $8.96 \mathrm{lg}$ $\mathrm{CFU} / \mathrm{g}$, respectively. At the end of stages, the total mesophilic microorganisms and the number of LAB gradually became stable. The total mesophilic microorganisms in $\mathrm{NF}$ and IF were $8.02 \mathrm{lg} \mathrm{CFU/g}$ and $8.53 \mathrm{lg} \mathrm{CFU} / \mathrm{g}$, respectively, and the number of LAB was $8.01 \mathrm{lg} C F U / \mathrm{g}$ and $8.45 \mathrm{lg} \mathrm{CFU} / \mathrm{g}$, respectively. It can be seen from the above results that the overall change trend of the total mesophilic microorganisms and the number of LAB in the two fermentation modes was similar. In addition, inoculation of LAB can increase the content of LAB in the fermented vegetables, accelerate the growth and reproduction rate, and increase the number of total mesophilic microorganisms in the fermented vegetables.

\section{Monitoring of the acidification process}

As shown in Fig. 2, the two fermentation modes had a $\mathrm{pH}$ of about 6.4 at the start of fermentation. In the initial stage of fermentation, the $\mathrm{pH}$ value decreased rapidly. In the subsequent fermentation process, with the prolongation of fermentation time, the nutrients in the fermented vegetables gradually decreased, the microbial metabolites gradually accumulated, and the $\mathrm{pH}$ decreased gradually. In the fermentation process of fermented vegetables, the total acid content of the fermented vegetables continuously increases with the prolongation of fermentation time. On the 4th day, for NF, the $\mathrm{pH}$ reached 3.71, the total acid content was $0.88 \mathrm{~g} / \mathrm{kg}$, and then slowly decreased to the $\mathrm{pH}$ was 3.44 , and the total acid content was $1.09 \mathrm{~g} / \mathrm{kg}$ at the end of the stage. For the IF, the
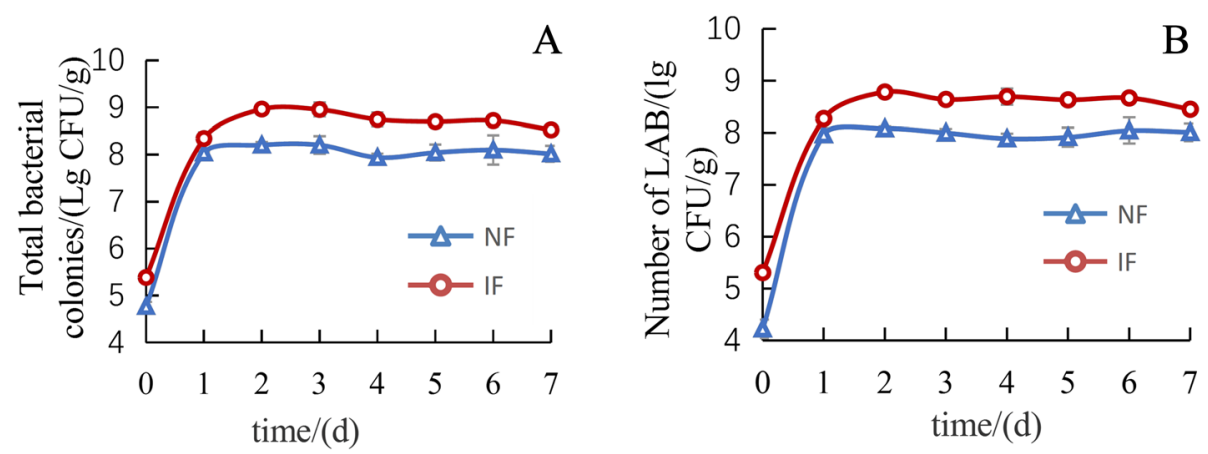

Fig. 1 Changes in microbial populations during vegetable fermentation. a Total mesophilic microorganisms counts. b Number of LAB. NF natural fermentation, IF inoculated fermentation 


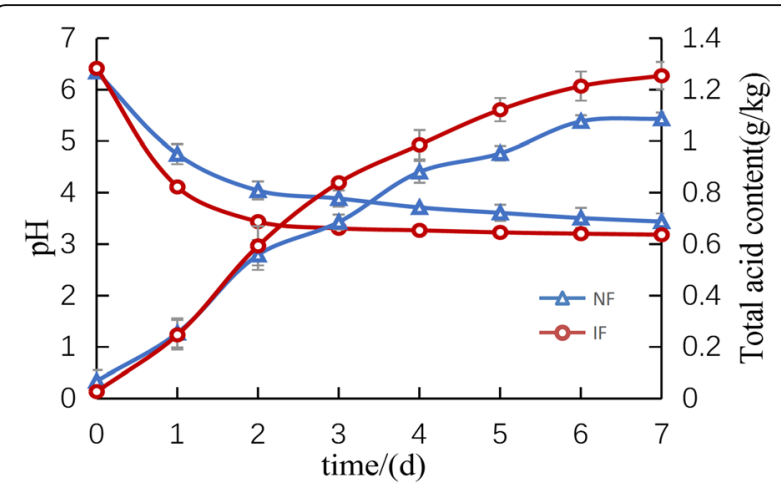

Fig. 2 Changes in $\mathrm{pH}$ and total acid content during vegetable fermentation. NF natural fermentation, IF inoculated fermentation

total acid content was $1.25 \mathrm{~g} / \mathrm{kg}$, and $\mathrm{pH}$ was 3.18 at the end of the stage. Studies had shown that the $\mathrm{pH}$ value of fermented vegetables was about 3.50 3.80 (Zhang et al. 2016), the IF has reached the $\mathrm{pH}$ of fermentation on the 2nd day, and the fermentation cycle of IF was significantly shorter than that of NF. It was consistent with previous research.

\section{Nitrite content changes in NF and IF}

In these fermented vegetables, nitrite levels were below $2.0 \mathrm{mg} / 100 \mathrm{~g}$ (calculated as sodium nitrite), a safe level was below the World Health Organization's Acceptable Daily Intake for a healthy adult $(0.06 \mathrm{mg} / \mathrm{kg}$ body weight per day $~ 3.6 \mathrm{mg}$ per day for a $60 \mathrm{~kg}$ person) (Ding et al. 2018). The production of nitrite in fermented vegetables was mainly due to the action of enzymes in the raw materials to reduce nitrates to nitrites. The nitrite content in the NF and the IF were increased and then decreased and the nitrite content of the IF was significantly lower than that of the NF (Fig. 3). A small "Nitrite peak" appeared on the first day of NF. The nitrite content reached $2.18 \mathrm{mg} / \mathrm{kg}$, which gradually decreased in the subsequent fermentation process, and then stabilized. For the IF, the content also reached the maximum value

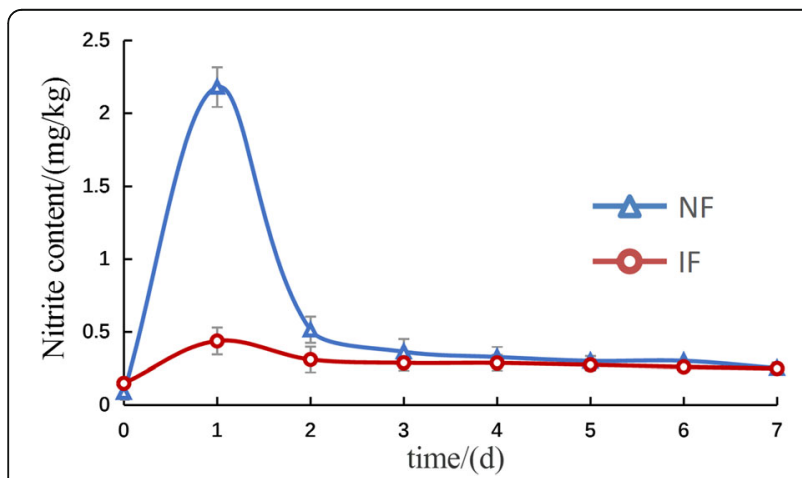

Fig. 3 Changes in Nitrate content during vegetable fermentation. NF natural fermentation, IF inoculated fermentation on the first day, which was $0.44 \mathrm{mg} / \mathrm{kg}$, and then gradually decreased, and it became stable at the end of fermentation. It was reported that in the early stage of NF, the content of LAB was less, and there were more other bacteria. The reductase produced by the bacteria can reduce the nitrate to nitrite, thus producing a "nitrite peak" (Guan et al. 2020). LAB can decompose nitrite and reduce the nitrite content in fermented vegetables, so the peak value of nitrite oxide in IF was significantly lower than that of NF.

\section{Volatile metabolite profiles determined by headspace SPME-GC/MS}

To understand the physical and chemical characteristics of fermented vegetables, SPME-GC/MS was used to analyze the volatile flavor substances in the products of NF and IF vegetables, including esters, acids, alcohols, alkenes, aldehydes, phenols, ketones, and benzene. There were 49 kinds of volatile flavor substances in NF vegetables and 63 kinds of volatile flavor substances in IF vegetables. In detail, twelve esters, seven alcohols, seven ketones, four benzenes, five alkenes, two phenols, eight aldehydes, and four acids were identified on the 7th day of NF; and thirteen esters, eleven alcohols, eight ketones, six benzenes, twelve alkenes, three phenols, six aldehydes, and four acids were identified on the 7th day of IF. Most of the volatile substances in the two fermentation methods were similar; among them, alcohols gave products a fresh smell, and were also the main source of esters. The relative content of alcohols in IF was $17.46 \%$, which was significantly higher than $14.29 \%$ of NF.

Variable importance in projection (VIP) is an index to measure the importance of independent variables in the O2PLS model. The VIP of 14 microorganism were demonstrated in Fig. 4. The increased VIP value indicated the more significant effect of microorganisms on volatile flavor substances. Lactobacillus was an important genus because it can use carbohydrates in vegetables to produce flavor substances. The final product of heterogeneous lactic acid fermentation was more abundant, which had a more significant impact on the flavor of fermented vegetables.

\section{Analysis of bacterial diversity NF and IF}

High-throughput 16S rDNA sequencing was performed on 28 samples, and 3,627,007 original sequences were obtained. After quality control, 3,202,325 sequences were obtained. The average coverage of the sample was 56,543. The sample sequence length was mostly concentrated on $430 \sim 450 \mathrm{bp}$. According to the sequence similarity level of $97 \%$, the samples were classified into operable classification units (OTUs). As shown in Table 1, the coverage of all samples was greater than 0.999 , indicating that the data obtained by this sequencing can truly reflect the actual 


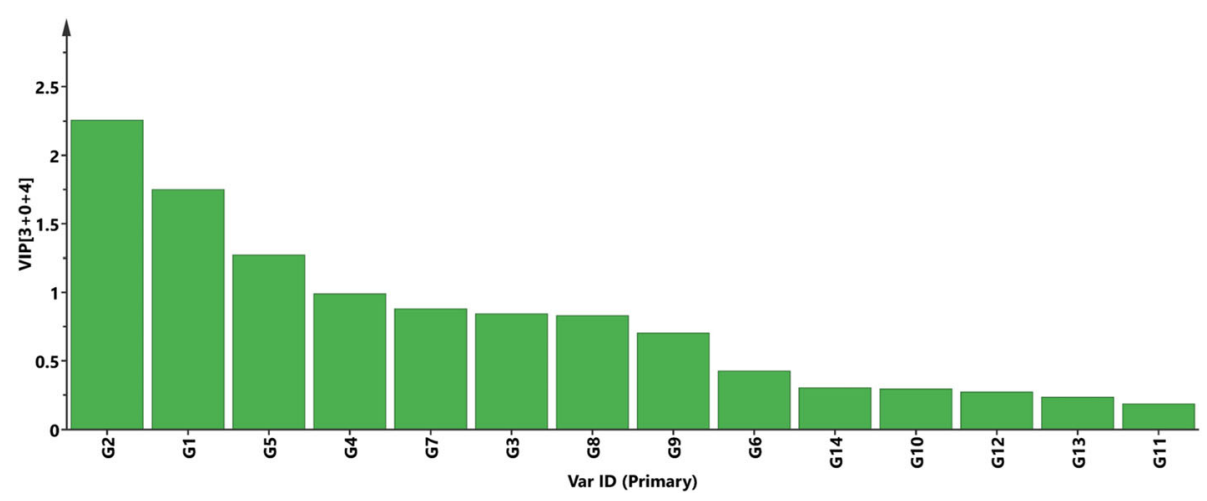

Fig. 4 VIP plot of the important microbiota correlated with flavors. G1: Cyanobacteria (norank); G2: Lactobacillus; G3: Leuconostoc; G4: Weissella; G5: Lactococcus; G6: Lactobacillales (unclassified); G7: Enterobacter; G8: Klebsiella; G9 Pectobacterium; G10: Enterobacteriaceae (unclassified); G11: Aeromonas; G12: Pantoea; G13: Acinetobacter; G14: Pseudomonas

situation of the samples. The amount of OTUs throughout the NF fermentation process was higher than that of IF. The OTUs and ACE indices were the highest at 0 day for both fermentation methods. Shannon and Simpson indicates the approximate number of species in the sample and the uniformity of its distribution, which shown opposite trends during the two fermentations. During the fermentation period of $0 \sim 7$ days, the Shannon and Simpson indices of NF and IF were between $55 \sim 71 \%$ and $36 \sim 45 \%$, respectively. The bacterial diversity and richness of NF were higher than that of IF.

Analysis of bacterial community composition in NF and IF The number of OTUs in four samples NF0, IF0, NF7 and IF7 was analyzed by Venn plot to evaluate the distribution of bacterial in the two different fermentation modes before and after fermentation. As shown in Fig. 5a, the bacterial diversity of NF vegetables was higher than that of IF. The relative abundance at the level of bacteria was shown in Fig. 5b. In the early stage of fermentation, due to the attachment of some bacteria to the surface of the vegetables, the species of the genus was more complicated, and the cyanobacteria account for a large proportion. The dominant genus in the NF process were Lactobacillus, Lactococcus, Weissella, Enterobacter, Klebsiella, Pectobacterium, Leuconostoc. Lactococcus, and Weissella were rapidly increased to $28.4 \%$ and $23.7 \%$ on the first day of fermentation, but gradually decreased during the fermentation. With the prolongation of fermentation time, Lactobacillus gradually became the dominant genus in NF vegetables, accounting for $53.4 \%$ on the 7 th day of fermentation, and $15.7 \%$ and $7.9 \%$ for Lactococcus and Weissella, respectively. During the IF process, the dominant genus was Lactobacillus. At the beginning of fermentation, Lactobacillus accounted for $18.3 \%$. On the 2 nd day, the growth rate of Lactobacillus reached the maximum, and the proportion continued to increase in the subsequent fermentation process. The proportion reached $90.2 \%$ on the 7 th day. The results showed that between the two fermentation methods, the Lactobacillus was the dominant one for the fermentation process. Lactobacillus can play an important role in the glycolysis pathway of the fermentation process, and the dominant species of NF was significantly more than the IF, indicating that IF can inhibit the growth

Table 1 Sequence abundance and microbial diversity in samples

\begin{tabular}{|c|c|c|c|c|c|c|c|c|c|c|c|c|}
\hline \multirow[t]{2}{*}{ Sample } & \multicolumn{2}{|c|}{ Average tags } & \multicolumn{2}{|c|}{ OTUs } & \multicolumn{2}{|c|}{ Shannon } & \multicolumn{2}{|c|}{ Simpson } & \multicolumn{2}{|l|}{ ACE } & \multicolumn{2}{|c|}{ Coverage } \\
\hline & NF & IF & NF & IF & NF & IF & NF & IF & NF & IF & NF & IF \\
\hline 0 day & 51,209 & 60,529 & 178 & 166 & 2.55 & 1.69 & 0.17 & 0.36 & 194.98 & 191.47 & 0.9995 & 0.9995 \\
\hline 1 days & 51,388 & 52,995 & 85 & 76 & 2.22 & 1.08 & 0.18 & 0.62 & 161.25 & 116.17 & 0.9995 & 0.9996 \\
\hline 2 days & 57,548 & 55,692 & 86 & 81 & 2.36 & 1.08 & 0.14 & 0.63 & 115.73 & 161.00 & 0.9997 & 0.9996 \\
\hline 3 days & 60,000 & 54,983 & 86 & 83 & 2.49 & 1.28 & 0.13 & 0.53 & 111.54 & 124.88 & 0.9996 & 0.9996 \\
\hline 4 days & 70,047 & 48,912 & 92 & 77 & 2.36 & 0.97 & 0.16 & 0.66 & 154.68 & 112.21 & 0.9997 & 0.9995 \\
\hline 5 days & 64,134 & 53,700 & 89 & 69 & 2.20 & 0.70 & 0.21 & 0.75 & 133.21 & 122.78 & 0.9997 & 0.9996 \\
\hline 6 days & 60,016 & 50,783 & 86 & 69 & 2.19 & 0.67 & 0.21 & 0.75 & 126.76 & 130.21 & 0.9996 & 0.9995 \\
\hline 7 days & 63,976 & 48,773 & 84 & 69 & 1.82 & 0.62 & 0.32 & 0.78 & 112.73 & 112.81 & 0.9997 & 0.9995 \\
\hline
\end{tabular}

NF natural fermentation, IF inoculated fermentation 


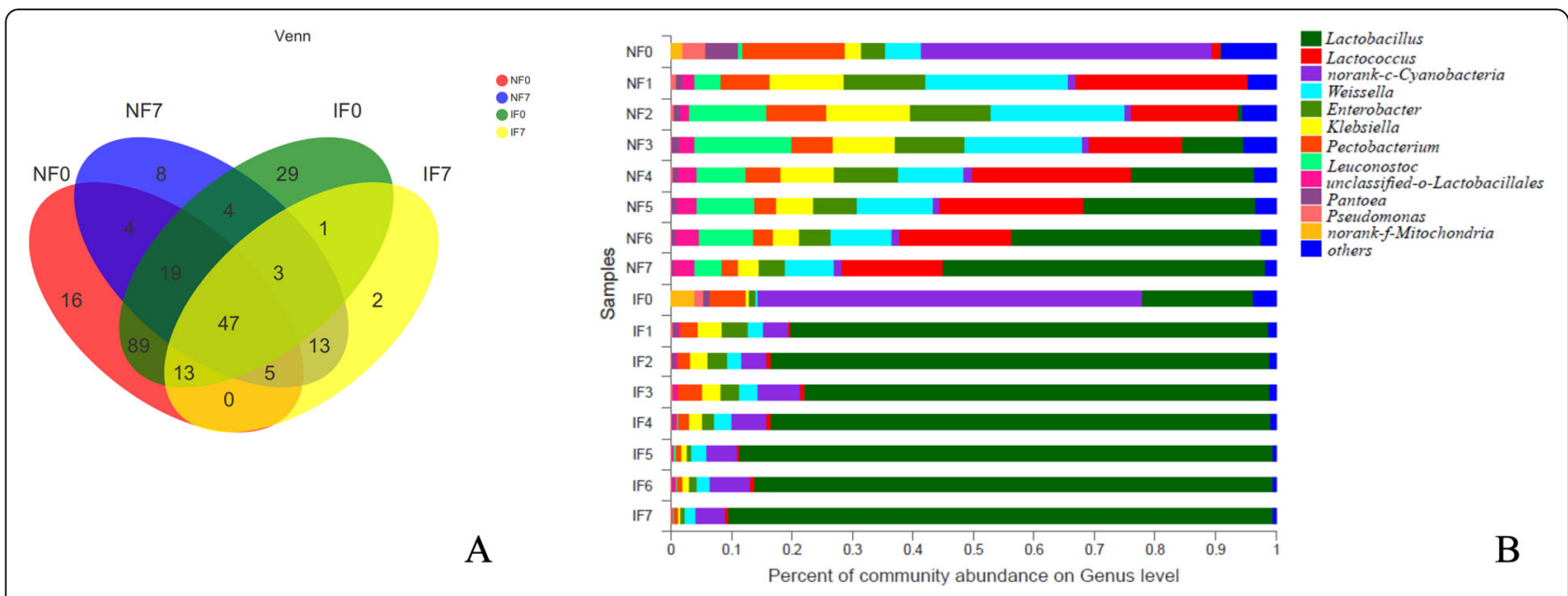

Fig. 5 Comparative analysis of bacterial community composition. a Analysis of flora structure of Veen. $\mathbf{b}$ Bacterial community composition. NF natural fermentation, IF inoculated fermentation; 0 7: days of fermentation

and reproduction of other microorganisms, so that fermented vegetables bacterial species were becoming more singular (Perez-Cataluna et al. 2018).

\section{Heat map analysis of NF and IF}

Four samples of NF0, IF0, NF7, and IF7 were analyzed by means of a heat map. The color gradients can be used to visually display the community differences of the samples and analyze the similarity of the samples. It can be seen from Fig. 6 that after the fermentation of the two fermentation modes, the genus of the abundance in the sample mainly included Lactobacillus, Lactococcus, Weissella, Enterobacter, Klebsiella, Pectobacterium, Leuconostoc, and Pseudomonas, etc. The distribution of bacteria in NF0 and IF0 was similar. The dominant genus of NF0 were Lactococcus and Pectobacterium. Lactobacillus was highly abundant in IF0 due to inoculation of LAB. The most abundant genus of NF7 were Lactobacillus and Lactococcus, and the dominant genus in IF7 was Lactobacillus.

It can be seen from the change of color depth that the content of bacteria in the IF was lower than that of NF. The initial, Stenotrophomonas, Sphingobacterium, Rhizobium, Flavobacterium, Saccharibacillus, Halomonas, Rheinheimera, Methylobacterium, etc. were mainly derived from soil and water. With the increase of fermentation time, the richness gradually was decreased in the IF samples, indicating that the IF can inhibit the growth and reproduction of some spoiled bacteria, retain only the dominant bacteria, increase the stability of the fermentation process, and improve the quality of fermented vegetables.

\section{Principal component analysis of NF and IF}

Principal component analysis (PCA) used the evolutionary information between sample sequences to calculate the sample distance. The closer the sample distribution points in the PCA diagram, the closer the bacterial diversity in the sample (Dubois et al. 2010). It can be observed from Fig. 7 that the first principal component (PC1) and the second principal component (PC2) can explain $96.6 \%$ of the original variable information. To a large extent, it can reflect the difference of bacterial diversity caused by the change of fermentation time under two different fermentation methods.

The NF process was divided into four clusters NF0; NF1, NF2, NF3; NF4; NF5, NF6, NF7. There were three advances, including two rapid shifts and one slower shift. The first rapid shift occurred in the first cluster to the second cluster of $\mathrm{PC} 2$, and the bacteria attached to the vegetables themselves gradually changed with the start of fermentation; the second rapid shift occurred in the negative correlation region of PC1, the second cluster to the third cluster, due to the gradual decrease of metabolite production during this fermentation, and the bacterial changes were also slowed down; the third slower shift occurred in the third cluster to the fourth cluster, the negative correlation region transfer to the positive correlation area indicated that the bacteria change tended to enter the late fermentation stage and the fermentation was basically completed.

IF can be divided into three clusters IF0; IF1, IF2, IF3; IF4, IF5, IF6, IF7. A total of two developments, including a rapid shift of $\mathrm{PC} 1$ and $\mathrm{PC} 2$, resulted in large changes due to the mutual influence of the bacteria carried by the vegetables and the inoculated $\mathrm{LAB}$. The other progress was a slower displacement, which was manifested in the positive correlation region of $\mathrm{PC} 1$, and the fermentation entered the late stage until the end of the fermentation process. 


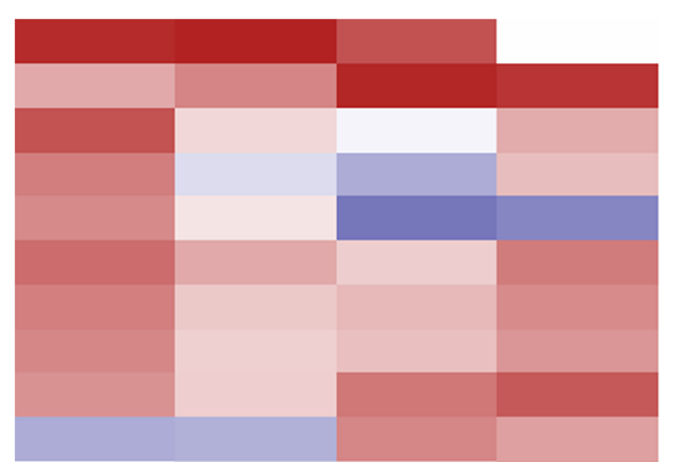

Lactobacillus

norank-c-Cyanobacteria

Lactococcus

Leuconostoc

unclassified-o-Lactobacillales

Weissella

Enterobacter

Klebsiella

Pectobacterium

norank-f-Mitochondria

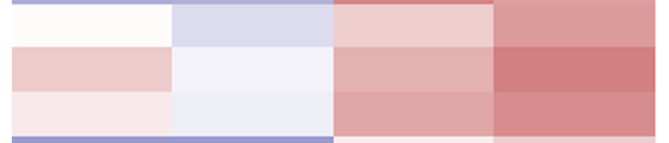

Acinetobacter

Pantoea

Pseudomonas

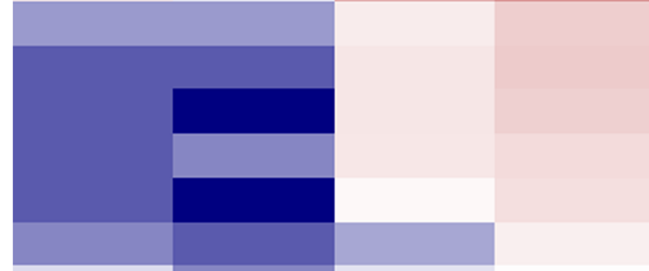

Stenotrophomonas

Sphingobacterium

Rhizobium

Flavobacterium

Chryseobacterium

Vogococcus

Dickeya

Comamonas

Saccharibacillus

Halomonas

Rheinheimera

Sphingobium

Methylobacterium

Cellvibrio

unclassified-f-Leuconostocaceae

Aeromonas

unclassified-f-Enterobacteriaceae

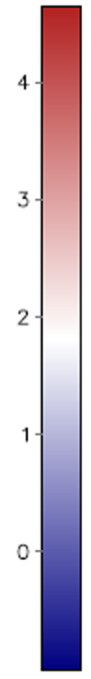

Fig. 6 Heat map of clustering during vegetable fermentation. NFO natural fermentation day 0, NF7 natural fermentation day 7, IF0 inoculated fermentation day 0 , IF7 inoculated fermentation day 7

\section{Correlation analysis of NF and IF}

The association between bacterial communities and $\mathrm{pH}$,

\section{total acid, nitrite}

At the bacterial genus level, the results of correlation analysis of $\mathrm{pH}$, total acid (TA), nitrite content $(\mathrm{N})$, and bacterial community of NF and IF vegetables were shown in the Fig. 8. RDA analysis explained a total of $46.43 \%$ of community changes, RDA1 explained $39.43 \%$, and RDA2 explained $7.00 \%$. pH $(P=0.001)$ was positively correlated with Norank-c-Cyanobacteria and Pectobacterium, and negatively correlated with Lactococcus and Lactobacillus. Total acid $(P=0.001)$ was positively correlated with Lactococcus and Lactobacillus; nitrite content $(P=0.008)$ with Norank-c-Cyanobacteria, Pectobacterium, and Lactococcus were positively correlated, and negatively correlated with Lactobacillus. It was obtained that Lactobacillus gradually increased to become a dominant genus in the vegetable fermentation process, which could significantly reduce $\mathrm{pH}$ and nitrite content.

The association between bacterial communities and flavors Bidirectional orthogonal partial least squares (O2PLS) (Bylesjö et al. 2007) was used to analyze the correlation between the bacterial and volatile flavor substances in the vegetable fermentation process. It included 14 bacterial and 64 volatile flavor substances, after modeled with SIMCA-P software; its $R^{2} \mathrm{X}=0.908>0.5$ showed that the model had a better interpretation. According to Pearson's method, the relevant indexes of $0.6-0.8$ was considered to indicate correlation (Rao et al. 2020). The results of the plot were shown in Fig. 9; a total of 11 volatile flavor substances showed correlation with LAB, where G1, G5, and G7 were correlated with one volatile flavor substance, respectively; G2, G3, G4, G8, and G10 showed correlation 


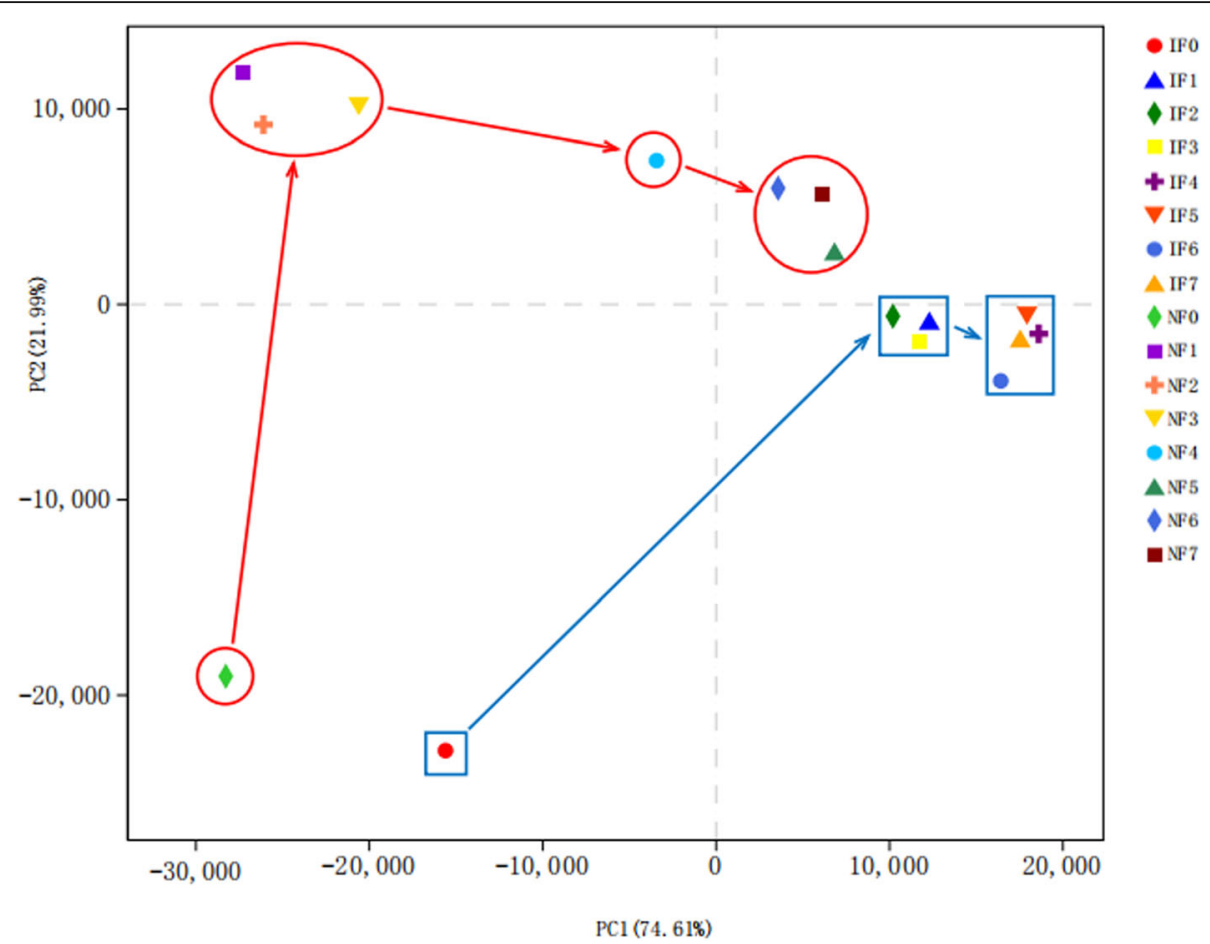

Fig. 7 Principal component analysis of bacterial community structure during vegetable fermentation. PC1 and PC2 explain $74.61 \%$ and $21.99 \%$ of variation, respectively. The statistics were performed based on Bray-Curtis distance. NF natural fermentation, IF inoculated fermentation; 0 7: days of fermentation

with two kinds of volatile flavor substances, respectively; G6 and G11 were correlated with three volatile flavor substances. Esters were one of the main characteristic flavors of fermented vegetables. Esters were positively correlated with bacteria, among which ethyl palmitate (E9) was positively correlated with four bacteria, namely G3, G4, G5, and G6 (Fig. 9). Our results showed that LAB played a vital role in the formation of fermented vegetable flavors.

\section{Discussion}

To determine whether there is a difference of the microbe biodiversity between NF and IF and understand

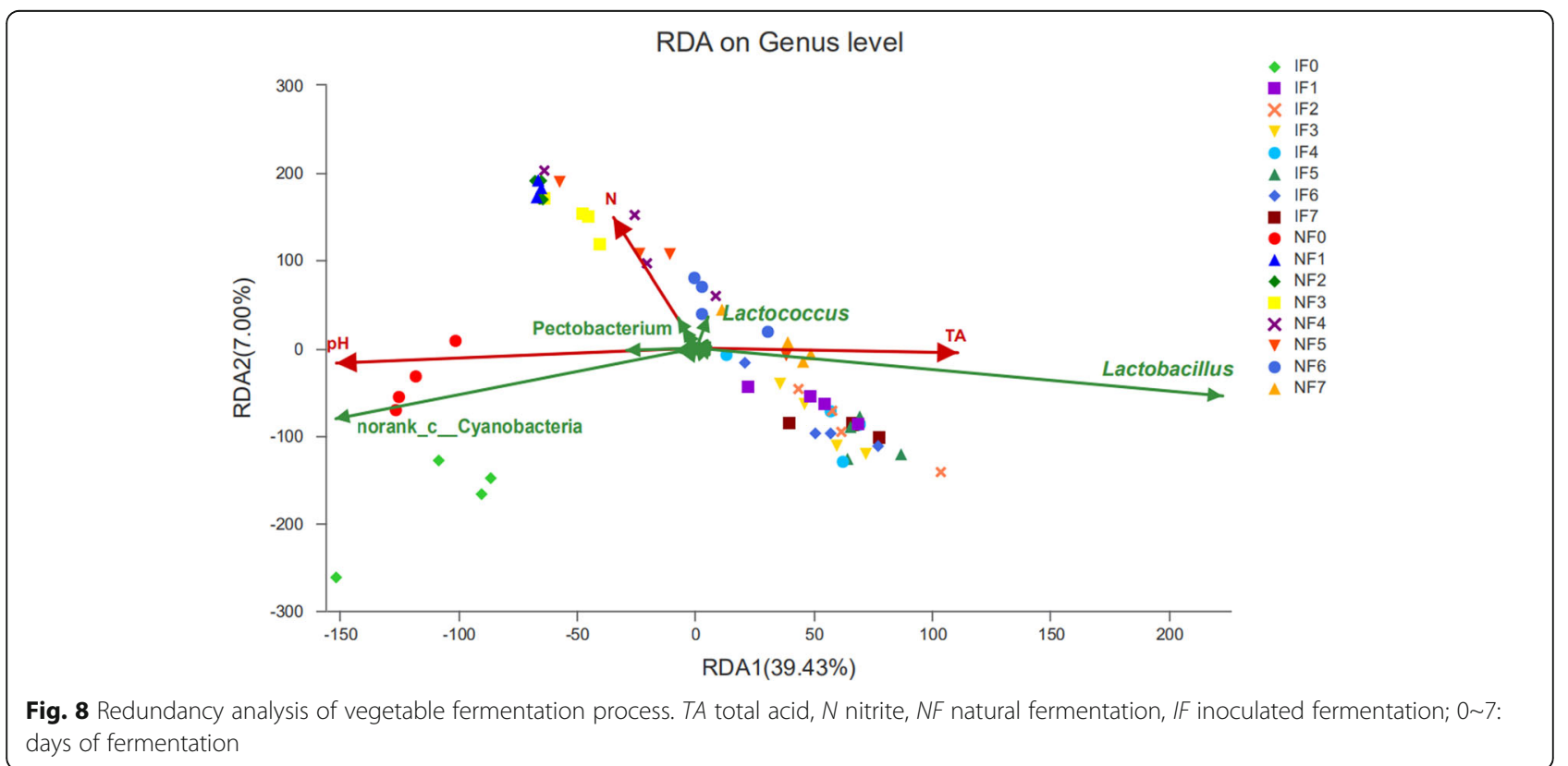


the fermentation process better, our study shows dramatic differences in the number of microorganisms, the bacterial diversity, and physicochemical indexes of Shanxi local fermented vegetables between NF and IF. We found changes in $\mathrm{pH}$, total acid content, and nitrate content during the fermentation process. The correlation analysis of $\mathrm{pH}$, total acid (TA), nitrite content $(\mathrm{N})$, and bacterial community was revealed by the redundancy analysis. There is a negative correlation between the LAB bacteria and flavors in the fermented vegetables.

Total mesophilic microorganisms and the number of LAB increased in the early stage and kept stable in the later stage. We detected that $\mathrm{LAB}$ to be dominant in the $\mathrm{NF}$ and IF. The number of LAB in IF was higher than that of NF. For the results of the bacterial community composition, we found that the diversity of the NF and IF gradually decreased with the extension of fermentation time, and the IF decreased more significantly.

As expected, in NF, the dominant bacterial genera were Lactobacillus and Weissella in the early stage of fermentation. Many studies showed that Lactobacillus, Lactococcus, and Weissella dominate during the fermentation of vegetables (Jeong et al. 2013, Young et al. 2014). Satoru et al. (2020) determined the bacterial community structure in 29 naturally fermented sunki samples through metagenomics analysis targeted by $16 \mathrm{~S}$ rRNA genes. The results showed that Lactobacillus was dominant in all samples and various bacterial species. Yang et al. (2018) revealed that the predominant bacteria in Chongqing radish kimchi brine were Lactobacillus,
Lactococcus, and Weberella. These studies were consistent with the results of this study.

The bacterial diversity of fermented vegetables of the NF and IF were characterized using Illumina MiSeq sequencing. The result was similar to the change of the LAB count. In both fermented vegetables, the dominant genus was LAB in the late fermentation period, consistent with Xiong et al. (2015). Also, previous research showed that the Lacticaseibacillus casei, Levilactobacillus brevis, and Lactiplantibacillus plantarum were detected in fermented vegetables (Yu et al. 2012). Through the Venn diagram, PCA, and heat map cluster analysis, we identified the differences in the bacterial flora structure between two fermentation methods.

The changes in $\mathrm{pH}$ and total acid as measures of the maturation time of fermented vegetables were as expected. During the fermentation process, the growth of microorganisms with lower acid and salt tolerance was gradually suppressed (Vethachai et al. 2007). The LAB in the IF quickly became the dominant bacterial group. This trend is consistent with previous reports in bacterial count and bacterial diversity analysis (Xiong et al. 2016; Wu et al. 2013). The OTU and Shannon index of bacterial communities in fermented vegetables correlated with the change of $\mathrm{pH}$ and total acid. There was no "nitrite peak" during the IF fermentation. IF fermentation was safer and more controlled than that of NF. It is known that LAB could reduce nitrite and improve food safety (Chen et al. 2014a; Tripathi and Giri 2014). IF inhibited the growth of mixed bacteria and decreased the content of reductase and the peak value of nitrite

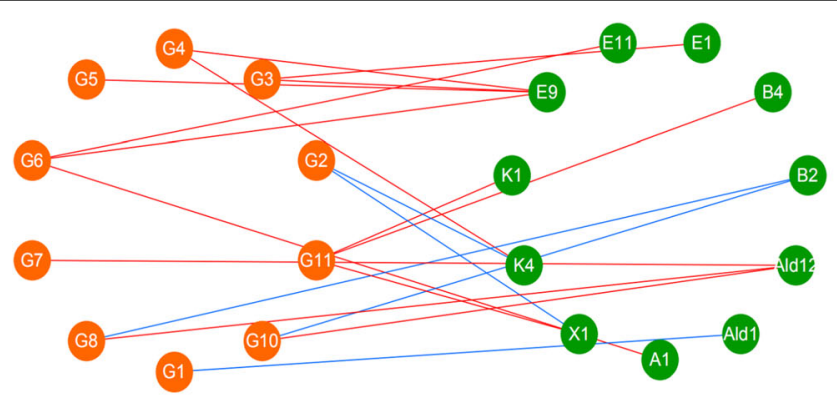

G1: Cyanobacteria (norank) G2: Lactobacillus G3: Leuconostoc G4: Weissella G5: Lactococcus G6: Lactobacillales (unclassified) G7: Enterobacter G8: Klebsiella G10: Enterobacteriaceae (unclassified) G11: Aeromonas
E1: Allyl isothiocyanate E9: Ethyl palmitate E11: (Z,Z,Z)-9,12,15-

Octadecatrienoic acid, ethyl ester $\mathrm{K} 1$ : $\mathrm{D}(+)$ Carvone K4: 4-[2,2,6trimethyl-7oxabicyclo[4.1.0]hept-1yl]-3-Buten-2-one B2: cis-Anethol B4: pisopropyltoluene $\mathrm{X} 1$ : aPhellandrene Ald1: Phenylacetaldehyde Ald12: Anisic aldehyde A1: Palmitic acid

Fig. 9 Correlation network between the bacterial and flavors of fermented vegetables during the $0 \sim 7$ days. $|\rho|>0.6$. The circle on the left represents bacteria that are generally associated with flavor. The circle on the right represents the different flavors associated with the bacterial community. The long red line connecting the circles indicates a positive correlation, and the blue indicates a negative correlation between bacterial community and flavor 
(Guan et al. 2020). Kim et al. (2017b) found that LAB can improve the nitrite removal ability of kimchi. The fermentation process tended to stabilize with the extension of the fermentation time by the data of total mesophilic microorganisms, $\mathrm{LAB}$, changes in $\mathrm{pH}$, and total acid.

There is a correlation between the bacterial and volatile flavor substances in the vegetable fermentation process based on our results. Volatile substances in fermented vegetables are mainly produced by the metabolism of raw and auxiliary materials and microorganisms of fermented vegetables. LAB played an important role in the fermentation process, especially Lactobacillus, Lactococcus, and Weissella were the dominant bacteria in flavor production. Lactococcus was positively correlated with ethyl palmitate (E9), whereas Weissella was positively correlated with ethyl palmitate (E9) and 4-[2,2, 6-trimethyl-7-oxabicyclo [4.1.0] hept-1-yl]-3-Buten-2one (K4). Lactobacillus had a negative correlation with 4-[2,2,6-trimethyl-7-oxabicyclo [4.1.0] hept-1-yl]-3Buten-2-one (K4) and a-Phellandrene (X1). However, Xiao et al. (2018) analyzed the correlation between bacterial and flavor substances in fermented pickles; Lactobacillus was correlated with 28 flavors, of which 13 flavors are negatively correlated. There was a difference between the current correlation between bacterial and flavor with his research results. This may be related to the selected raw materials and fermentation methods. Notably, some associations were revealed based on the statistical correlations.

In summary, this study shows higher biodiversity of bacteria in the natural fermentation process than the inoculated fermentation process. It was interesting to see the difference of nitrite change between two fermentation methods, indicating different dynamics of metabolism between two groups of microbiomes. Based on changes of $\mathrm{pH}$, total acid, and nitrite, the inoculated fermentation seems to be safer and the product quality is more stable. LAB were the dominant strain in the inoculated fermentation process as expected. Profiles of volatile flavor substances compared between NF and IF were revealed. Overall, we have provided the novel scientific knowledge in the bacteria biodiversity and how they affect the taste and flavor, which is the foundation for food processing industry to form a standardization for the vegetable fermentation process.

\section{Conclusions}

The diversity of bacteria in naturally fermented vegetables was more enriched than that in inoculated fermentation. The total number of mesophilic microorganisms and lactic acid bacteria in inoculated fermentation was dominant. However, inoculation fermentation can shorten the time of the fermentation cycle and reduce nitrite content, which can provide a theoretical basis for the standardized production of traditional fermented vegetables.

\section{Acknowledgements}

The authors are grateful to Dr. Min Zhang from the Beijing Technology and Business University for her assistance in revising the first draft of the article.

\section{Authors' contributions}

ZC conducted research on correlation analysis and drafted a manuscript. JK designed the research and carried out the experiments. $Y Z$ participated in the experiments. XP participated in the data analysis. XY participated in its design and coordination. HL-B improved the writing of the manuscript. XG designed the experimental scheme, and carried out the overall planning and improvement of the manuscript. All authors have read and approved the final manuscript.

\section{Funding}

This work was funded by the Beijing Leafy Vegetables Innovation Team of Modern Agro-industry Technology Research System (BAIC07-2020), Research Fund for Academic Degree \& Graduate Education of Beijing University of Agriculture (2020YJS033), and Beijing Municipal Agriculture Committee's new-type production and operation main body technology capacity improvement project (26).

\section{Availability of data and materials}

The datasets used and/or analyzed during the current study are available from the corresponding author on reasonable request.

Ethics approval and consent to participate

Not applicable.

Consent for publication

Not applicable.

\section{Competing interests}

The authors declare that there is no competing interest.

\section{Author details}

${ }^{1}$ Beijing Laboratory of Food Quality and Safety, Beijing Key Laboratory of Agricultural Product Detection and Control of Spoilage Organisms and Pesticide Residue, Beijing Engineering Laboratory of Probiotics Key Technology Development, Beijing University of Agriculture, Beijing 102206, China. ${ }^{2}$ Beijing Dongcheng District Youth Lake Primary School, Beijing 100011, China. ${ }^{3}$ Agricultural Research and Development Program, Central State University, Wilberforce, $\mathrm{OH}$ 45305, USA.

Received: 4 August 2020 Accepted: 3 November 2020 Published online: 25 November 2020

\section{References}

Bylesjö M, Eriksson D, Kusano M, Moritz T, Trygg J (2007) Data integration in plant biology: the O2PLS method for combined modeling of transcript and metabolite data. Plant J 52:1181-1191

Caggianiello G, Kleerebezem M, Spano G (2016) Exopolysaccharides produced by lactic acid bacteria: from health-promoting benefits to stress tolerance mechanisms. Appl Microbiol Biotechnol 100:3877-3886

Chen C, Chen X, Jiang M, Rui X, Li W, Dong M (2014a) A newly discovered bacteriocin from Weissella hellenica D1501 associated with Chinese Dong fermented meat (Nanx Wudl). Food Control 42:116-124

Chen G, Yu W, Zhang Q, Song P, Zhang B, Liu Z et al (2014b) Research of sichuan paocai and lactic acid bacteria. Advance J Food Sci Technol 6:1-5

Ding Z, Suzanne D. J., Robert Price, Rong Reynolds, Truong V, Summer Conley Payton et al (2018) Evaluation of nitrate and nitrite contents in pickled fruit and vegetable products. Food Control 90: 304-311.

Dubois P, Trynka G, Franke L, Hunt K, Romanos J, Curtotti A et al (2010) Multiple common variants for celiac disease influencing immune gene expression. Nat Genet 42:295-302

Guan Q, Zheng W, Huang T, Xiao Y, Liu Z, Peng Z et al (2020) Comparison of microbial communities and physiochemical characteristics of two traditionally fermented vegetables. Food Res Int:128 
Han B, Cao C, Rombouts FM, Nout Robet MJ (2004) Microbial changes during the production of Sufu--a Chinese fermented soybean food. Food Control 15: 265-270

He Z, Chen H, Wang X, Lin X, Ji C, Li S et al (2020) Effects of different temperatures on bacterial diversity and volatile flavor compounds during the fermentation of suancai, a traditional fermented vegetable food from northeastern China. Lwt-Food Sci Technol 118:108773

Jampaphaeng K, Ferrocino I, Giordano M, Rantsiou K, Maneerat S, Cocolin L (2018) Microbiota dynamics and volatilome profile during stink bean fermentation (Sataw-Dong) with Lactobacillus plantarum KJ03 as a starter culture. Food Microbiol 76:91-102

Jeong S, Lee H, Jung J, Lee S, Seo H, Park W et al (2013) Effects of red pepper powder on microbial communities and metabolites during kimchi fermentation. Int J Food Microbiol 160:252-259

Jung JY, Lee SH, Jeon CO (2014a) Kimchi microflora: history, current status, and perspectives for industrial kimchi production. Appl Microbiol Biotechnol 98 : 2385-2393

Jung JY, Lee SH, Kim JM, Park MS, Bae JW, Hahn Y et al (2011) Metagenomic analysis of kimchi, a traditional Korean fermented food. Appl Environ Microbiol 77:2264-2274

Jung JY, Lee SH, Lee HJ, Seo HY, Park WS, Jeon CO (2012) Effects of Leuconostoc mesenteroides starter cultures on microbial communities and metabolites during kimchi fermentation. Int J Food Microbiol 153:378-387

Jung MY, Kim TW, Lee C, Kim JY, Song HS, Kim YB et al (2018) Role of jeotgal, a Korean traditional fermented fish sauce, in microbial dynamics and metabolite profiles during kimchi fermentation. Food Chem 265:135-143

Jung SJ, Kim MJ, Chae SW (2016) Quality and functional characteristics of kimchi made with organically cultivated young Chinese cabbage ( olgari-baechu ). J Ethnic Foods 3:150-158

Jung SJ, Park SH, Choi EK, Cha YS, Cho BH, Kim YG et al (2014b) Beneficial effects of Korean traditional diets in hypertensive and type 2 diabetic patients. J Med Food 17:161-171

Khan KA, Gulab H, Haider F (2018) Method development for the determination of commercial formulation of difenoconazole fungicide using uv-visible spectrophotometer. J Anal Bioanal Tech 09:1000410-1000416

Kim EK, An SY, Lee MS, Kim TH, Lee HK, Hwang WS et al (2011) Fermented kimchi reduces body weight and improves metabolic parameters in overweight and obese patients. Nutr Res 31:436-443

Kim M, Kim K (2012) Isolation and Identification of $y$-Aminobutyric acid (GABA)producing lactic acid bacteria from Kimchi. Journal of the Korean Society for Applied Biological Chemistry 55:777-785

Kim MJ, Lee HW, Lee ME, Roh SW, Kim TW (2019) Mixed starter of Lactococcus lactis and Leuconostoc citreum for extending kimchi shelf-life. J. Microbiol. 57: 479-484

Kim SH, Kang KH, Kim SH, Lee S, Lee SH, Ha ES et al (2017a) Lactic acid bacteria directly degrade $\mathrm{N}$-nitrosodimethylamine and increase the nitrite-scavenging ability in kimchi. Food Control 71:101-109

Kim SS, Park KJ, An HJ, Choi YH (2017b) Phytochemical, antioxidant, and antibacterial activities of fermented Citrus unshiu byproduct. Food Sci. Biotechnol. 26:461-466

Kwak CS, Park SC, Song KY (2012) Doenjang, a fermented soybean paste, decreased visceral fat accumulation and adipocyte size in rats fed with high fat diet more effectively than nonfermented soybeans. J Med Food 15:1-9

Lee $M$, Song JH, Jung M, Lee SH, Chang JY (2017) Large-scale targeted metagenomics analysis of bacterial ecological changes in 88 kimchi samples during fermentation. Food Microbiol 66:173-183

Lee SH, Jung JY, Jeon CO (2015a) Source tracking and succession of kimchi lactic acid bacteria during fermentation. J Food Sci 80:1871-1877

Lee YC, Kung HF, Huang YL, Wu CH, Huang YR, Tsai YH (2016) Reduction of biogenic amines during miso fermentation by lactobacillus plantarum as a starter culture. J Food Prot 79:1556-1561

Lee YC, Lin CS, Liu FL, Huang TC, Tsai YH (2015b) Degradation of histamine by Bacillus polymyxa isolated from salted fish products. J Food Drug Anal 23: 836-844

Mcfeeters RF (2004) Fermentation microorganisms and flavor changes in fermented foods. J Food Sci 69:35-37

Park EJ, Chun J, Cha CJ, Park WS, Jeon CO, Bae JW (2012) Bacterial community analysis during fermentation of ten representative kinds of kimchi with barcoded pyrosequencing. Food Microbiol 30:197-204
Perez-Cataluna A, Elizaquivel P, Carrasco P, Espinosa J, Reyes D, Wacher C et al (2018) Diversity and dynamics of lactic acid bacteria in Atole agrio, a traditional maize-based fermented beverage from South-Eastern Mexico, analysed by high throughput sequencing and culturing. Antonie Van Leeuwenhoek 111:385-399

Rao Y, Tao Y, Chen X, She X, Qian Y, Li Y et al (2020) The characteristics and correlation of the microbial communities and flavors in traditionally pickled radishes. Lwt-Food Sci Technol 118:108804

Satoru T, Jun W, Toshihide N, Akihito E, Sanae O (2020) Characterisation of the bacterial community structures of sunki, a traditional unsalted pickle of fermented turnip leaves. J Biosci Bioeng 129:541-551

Tamang JP, Watanabe K, Holzapfel WH (2016) Review: diversity of microorganisms in global fermented foods and beverages. Front Microbiol 7: 377

Tripathi M, Giri S (2014) Probiotic functional foods: survival of probiotics during processing and storage. J Funct Foods 9:225-241

Vethachai P, Fredrick B, Lu ZJ, P. FH (2007) DNA fingerprinting of lactic acid bacteria in sauerkraut fermentations. Appl Environ Microbiol 73: 7697-7702.

Wei B, Peng Z, Huang T, Guan Q, Xie M, Xiong T (2020) Stability of potential prophages in commercial strain Lactobacillus plantarum NCU116 under various stressors. Arch Microbiol 202:1241-1250

Wu R, Wu Z, Zhao C, Lv C, Wu J, Meng X (2013) Identification of lactic acid bacteria in suancai, a traditional Northeastern Chinese fermented food, and salt response of Lactobacillus paracasei LN-1. Ann Microbiol 64:1325-1332

Xiang W, Zhang N, Lu Y, Zhao Q, Xu Q, Rao Y et al (2020) Effect of Weissella cibaria co-inoculation on the quality of Sichuan Pickle fermented by Lactobacillus plantarum. Lwt-Food Sci Technol:121. https://doi.org/10.1016/j. Iwt.2019.108975

Xiao Y, Xiong T, Peng Z, Liu C, Huang T, Yu H et al (2018) Correlation between microbiota and flavours in fermentation of Chinese Sichuan Paocai. Food Res Int 114:123-132

Xiong T, Li J, Liang F, Wang Y, Guan Q, Xie M (2016) Effects of salt concentration on Chinese sauerkraut fermentation. LWT - Food Sci Technol 69:169-174

Xiong T, Peng F, Li X, Li J, Qianqian G (2015) Dominant microorganisms and their metabolic characteristics of traditional fermented kimchi. Food Sci 36:158161

Xiong T, Song SH, Huang XH, Feng C, Liu GQ, Huang JQ et al (2013) Screening and identification of functional lactobacillus specific for vegetable fermentation. J Food Sci 78:84-89

Yang J, Cao J, Xu H, Hou Q, Yu Z, Zhang H et al (2018) Bacterial diversity and community structure in Chongqing radish paocai brines revealed using PacBio single-molecule real-time sequencing technology. J Sci Food Agric 98:3234-3245

Yang X, Hu W, Xiu Z, Jiang A, Yang X, Saren G et al (2020) Microbial community dynamics and metabolome changes during spontaneous fermentation of northeast sauerkraut from different households. Front Microbiol 11

Yeh CT, Yen GC (2005) Effect of sulforaphane on metallothionein expression and induction of apoptosis in human hepatoma HepG2 cells. Carcinogenesis 26: $2138-2148$

Young JJ, Hee LS, Ok JC (2014) Kimchi microflora: history, current status, and perspectives for industrial kimchi production. Appl Microbiol Biotechnol 98 2385-2393

Yu J, Gao W, Qing M, Sun Z, Wang W, Liu W et al (2012) Identification and characterization of lactic acid bacteria isolated from traditional pickles in Sichuan, China. J Gen Appl Microbiol 58:163-172

Zhang Q, Chen G, Wenxi S, Wang Y, Zhang W, Chi Y (2016) Microbial safety and sensory quality of instant low-salt Chinese paocai. Food Control 59:575-580

Zhang ZP, Ma J, He YY, Lu J, Ren DF (2018) Antioxidant and hypoglycemic effects of Diospyros lotus fruit fermented with Microbacterium flavum and Lactobacillus plantarum. J Biosci Bioeng 125:1-6

\section{Publisher's Note}

Springer Nature remains neutral with regard to jurisdictional claims in published maps and institutional affiliations. 